Pre-print Manuscript of Article:

Zhang, Z., Sun, C., Bridgelall, R., Sun, M., "Road Profile Reconstruction Using Connected Vehicle

Responses and Wavelet Analysis," Journal of Terramechanics, DOI: 10.1016/j.jterra.2018.10.004, Vol. 80,

December 2018, Pages 21-30.

\title{
Road Profile Reconstruction Using Connected Vehicle Responses and Wavelet Analysis
}

\author{
Zhiming Zhang', Chao Sun ${ }^{2 *}$, Raj Bridgelall ${ }^{3}$, and Mingxuan Sun ${ }^{4}$ \\ ${ }^{1}$ Department of Civil and Environmental Engineering, Louisiana State University \\ 3325 Patrick F. Taylor, Baton Rouge, LA 70803, USA.zzhan59@lsu.edu \\ ${ }^{2 *}$ Corresponding author: Department of Civil and Environmental Engineering, Louisiana \\ State University, 3325 Patrick F. Taylor, Baton Rouge, LA 70803, USA. csun@lsu.edu \\ ${ }^{3}$ Upper Great Plains Transportation Institute, North Dakota State University \\ 1340 Administration Ave., Fargo, ND 58108, USA. raj.bridgelall@ndsu.edu \\ ${ }^{4}$ Computer Science and Engineering Division, School of Electrical Engineering and \\ Computer Science, Louisiana State University, $102 \mathrm{~F}$ Electrical Engineering Building, \\ Baton Rouge,LA 70803,USA.msun@csc.lsu.edu
}

\begin{abstract}
Practitioners analyze the elevation profile of a roadway to detect localized defects and to produce the international roughness index. The prevailing method of measuring road profiles uses a specially instrumented vehicle and trained technicians, which usually leads to a high cost and an insufficient measurement frequency. The recent availability of probe data from connected vehicles provides a method that is cost-effective, continuous, and covers the entire roadway network. However, no method currently exists that can reproduce the elevation profile from multi-resolution features of the vehicle inertial response signal. This research uses the wavelet decomposition of the vehicle inertial
\end{abstract}


responses and a nonlinear autoregressive artificial neural network with exogenous inputs to reconstruct the elevation profile. The vehicle inertial responses are a function of both the vehicle suspension characteristics and its speed. Therefore, the authors normalized the vehicle response models by the traveling speed and then numerically solved their inertial response equations to simulate the vehicle dynamic responses. The results demonstrate that applying the artificial neural network to the wavelet decomposed inertial response signals provides an effective estimation of the road profile.

Keywords: road roughness, profile reconstruction, vehicle response, wavelet analysis, neural network

\section{Introduction}

As pavement roughness accelerates vehicle wear, affects ride quality and transportation safety, expedites pavement deterioration, and increases fuel consumption, it has long been used as a major criteria to assess the road condition and guide the road maintenance and rehabilitation (Zhang, Deng et al. 2015, Kong, Abdullah et al. 2017, Qin, Wang et al. 2019). The prevailing method of roughness measurement uses a vehicle instrumented with an inertial profiler to collect road profile data (Spangler and Kelly 1966). The inertial profiler collects the road profile information at a highway speed and a sampling rate sufficient for further analysis of the profile's spectral composition. Despite its worldwide popularity, the profiling method has high cost and labor intensity that prevents agencies from measuring many roads more than once per year. As a result, the decisions on road maintenance and rehabilitation are usually made using outdated road roughness information. Moreover, infrequent road condition monitoring precludes the detection of severe immediate road distresses such as the frost heaves that occurs and vanishes within a year. This situation leads to information gaps of roadway safety and thus increases the liability of administrative bureaus (Zhang, Deng et al. 2016). 
The response-type method of roughness evaluation uses the dynamic responses of a probe vehicle traversing roughness roads, including the displacements, velocities, and accelerations of multiple components of the vehicle, to assess the road roughness severity indirectly. Compared with inertial profiling, the response-type method is less expensive because it reduces labor and equipment costs. Hence, this method allows more frequent and timely measurement and evaluation of road roughness conditions by agencies. Mostly, the response-type method yields an indirect roughness evaluation index through statistical analysis and signal processing on the collected data of vehicle responses, which is usually comparable to the widely used international roughness index (IRI) (Bridgelall 2014, Bridgelall, Huang et al. 2016). Recently, the response-type method has also been used to estimate the road profile by analyzing the collected data using advanced signal processing and system identification techniques (Ngwangwa, Heyns et al. 2010, Kong, Omar et al. 2014, Qin, Xiang et al. 2018). Literature review finds that there is limited research on road profile estimation using probe vehicle responses. All the existing studies use the collected signal series as the input to their estimating algorithms without discriminating its frequency composition. Vehicle responses, the output of a vehicle under the roughness excitation when travelling across a road section, reflects the road information as well as the vehicle characteristics, which compromises its representation of the roughness information if without appropriate signal decomposition and filtering.

Wavelet analysis provides a multi-resolution decomposition of a signal in the time or spatial domain. It is widely used for road profile and pavement surface analysis and evaluation. Qin et al. used wavelet analysis to extract features from vehicle responses for road roughness classification (Qin, Xiang et al. 2017). Wei and Fwa characterized road roughness using the wavelet energy statistics developed from a wavelet transform and found that it has a high correlation with IRI for both asphalt and concrete pavements (Wei and Fwa 2004). De Pont and Scott used wavelet analysis to find local roughness features by comparing the magnitude of a certain component with a reference value at that decomposition level (De Pont and Scott 1999). Papagiannakis et al. used wavelet analysis to decompose the pavement roughness and a truck's dynamic axle load and compared their relative energy within different wavelet sub-bands (Papagiannakis, 
Zelelew et al. 2007, Papagiannakis, Zelelew et al. 2007). Shokouhi et al. used wavelet-based multiresolution decomposition as a diagnostic tool to determine the location and extent of various frequency features of the road profile including pavement defects and surface anomalies (Shokouhi, Gucunski et al. 2005). Wei et al. used wavelet analysis as a tool to provide in-depth insights into the road profiles including the correlation of the IRI with the energy within the sub-bands, the energy distribution difference for profiles with close values of IRI, the effect of local features on IRI, and the identification of patterns of pavement roughness deterioration (Wei, Fwa et al. 2005). Zhou et al. used wavelet transform together with Radon transform to analyze pavement images for the purpose of pavement distress classification (Zhou, Huang et al. 2005).

Daubechies' wavelet family is widely used for signal processing due to their ease of implementation and their orthogonality that yields stable mathematical behaviors. Among the Daubechies' wavelet family, DB3 is the most widely used wavelet for road profile analysis (Wei and Fwa 2004, Wei, Fwa et al. 2005, Papagiannakis, Zelelew et al. 2007, Papagiannakis, Zelelew et al. 2007) because of its good resolution in both the spatial and frequency domains as well as its orthogonality and maximal flatness. These are desirable characteristics to support iterative decomposition in discrete wavelet transforms (Daubechies 1988). Researchers also use other wavelets from the Daubechies' family to analyze road roughness such as DB4 (De Pont and Scott 1999) and DB6 (Shokouhi, Gucunski et al. 2005).

Machine learning technique is an effective tool for data analysis and information extraction. It has been widely used to assess road condition by processing and analyzing the data collected from connected vehicles. Ngwangwa et al. used a Bayesian-regularized nonlinear autoregressive exogenous model (NARX) neural network to reconstruct road profiles (Ngwangwa, Heyns et al. 2010). Qin et al. used a probabilistic neural network (PNN) classifier to determine the road roughness class for a semi-active suspension system (Qin, Xiang et al. 2017). Guarneri et al. used a recurrent neural network to predict the dynamic behavior of vehicles traversing road irregularities by training the network with outputs from a simulated tire-suspension model (Guarneri, Rocca et al. 2008). Attoh-Okine aimed at predicting the IRI and conducted a detailed 
study on the influence of momentum-term and learning rate in the back-propagation algorithm (AttohOkine 1999). Tai et al. detected road anomalies, such as potholes and rutting, by processing the data collected from a motorcycle-mounted tri-axial accelerometer using the support vector machine (Tai, Chan et al. 2010). Hoffmann et al. developed an online road roughness classification system using bicycles instrumented with smartphones imbedded with the Naive Bayes algorithm (Hoffmann, Mock et al. 2013). Ward and Iagnemma used support vector machines to process the measured acceleration signal from a passenger vehicle to classify terrains after removing the impulses from anomalies including ruts and potholes (Ward and Iagnemma 2009).

Practitioners use the elevation profile data to characterize network-level roughness, estimate the spatial wavelength composition of a road segment, localize anomalies, and forecast maintenance needs. Therefore, the availability of roadway elevation profiles from widely available connected vehicle data sources will provide transportation agencies and users with efficient means to collect road condition data continuously, and network-wide. The literature review indicates a lack of research that addresses the estimation of a road elevation profile from connected vehicle data. This research contributes to this field by applying the NARX artificial neural network to the wavelet decomposed inertial responses from connected vehicles to estimate the road elevation profile. The organization of the paper is as follows: Section 2 describes the mathematical models used for the roughness generation and the vehicle response analysis. Section 3 describes the wavelet decomposition of the normalized vehicle responses that serve as inputs to the NARX network of road profile reconstruction. Section 4 reconstructs the road profiles by applying the NARX network to the wavelet decomposed signal components. Finally, Section 5 presents the conclusions and outlines the future work. 


\section{Model Description}

\subsection{Vehicle Roughness Interaction}

Figure 1 illustrates the dynamic model of a vehicle and a single dimension road profile. The vehicle model is a quarter car that contains a sprung mass $m_{\mathrm{s}}$, an un-sprung mass $m_{\mathrm{u}}$, a suspension spring $k_{\mathrm{s}}$, a tire spring $k_{\mathrm{u}}$, a suspension damper $c_{\mathrm{s}}$, and a tire damper $c_{\mathrm{u}}$. Studies of vehicle dynamics and ride quality use the quarter car model extensively because of its simplicity and effectiveness [29-32]. IRI is defined as the integrated relative difference between the sprung mass velocity and the un-sprung mass velocity over the distance traveled, assuming the model travels at a simulated speed of $80 \mathrm{~km} / \mathrm{h}$ over the measured road profile with roughness (Sayers 1995). In particular, IRI is a function of the sprung and un-sprung mass motions of a special quarter car that the international standard specifies to represent all vehicles, regardless of their actual parameter equivalents.

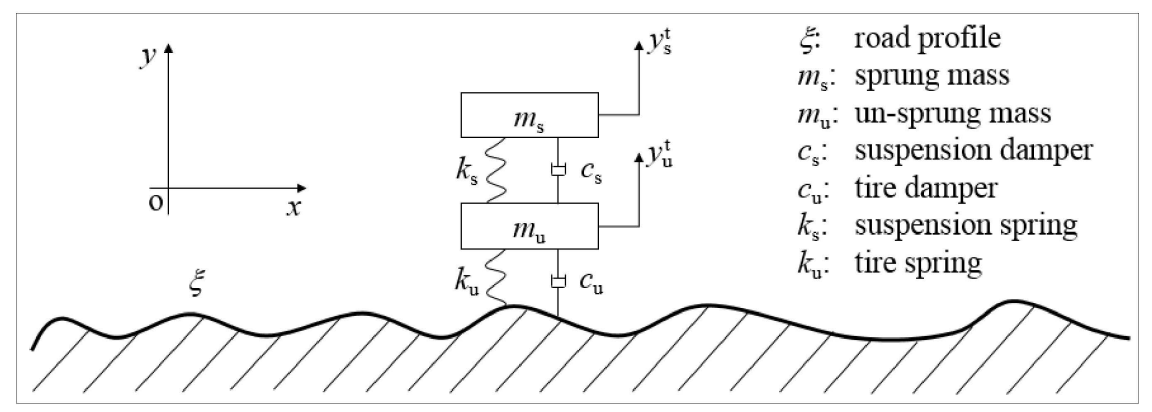

Figure 1. Dynamic model of the vehicle on a road profile

Suppose that $y_{\mathrm{s}}^{\mathrm{t}}$ and $y_{\mathrm{u}}^{\mathrm{t}}$ are the absolute displacements of the sprung and un-sprung masses, respectively. Let $y_{\mathrm{s}}$ and $y_{\mathrm{u}}$ denote the relative displacement of the sprung mass and the un-sprung mass, respectively, such that

$$
y_{\mathrm{s}}=y_{\mathrm{s}}^{\mathrm{t}}-y_{\mathrm{u}}^{\mathrm{t}} \text { and } y_{\mathrm{u}}=y_{\mathrm{u}}^{\mathrm{t}}-\xi
$$

Expressing the equations of motion of the dynamic system in the matrix form yields [33]: 


$$
M \ddot{Y}(t)+C \dot{Y}(t)+K Y(t)=R(t)
$$

where $\quad M=\left[\begin{array}{cc}m_{\mathrm{s}} & m_{\mathrm{s}} \\ 0 & m_{\mathrm{u}}\end{array}\right], \quad C=\left[\begin{array}{cc}c_{\mathrm{s}} & 0 \\ -c_{\mathrm{s}} & c_{\mathrm{u}}\end{array}\right], \quad K=\left[\begin{array}{cc}k_{\mathrm{s}} & 0 \\ -k_{\mathrm{s}} & k_{\mathrm{u}}\end{array}\right], \quad Y=\left\{\begin{array}{l}y_{\mathrm{s}} \\ y_{\mathrm{u}}\end{array}\right\}, \quad \dot{Y}=\left\{\begin{array}{l}\dot{y}_{\mathrm{s}} \\ \dot{y}_{\mathrm{u}}\end{array}\right\}, \quad \ddot{Y}=\left\{\begin{array}{l}\ddot{y}_{\mathrm{s}} \\ \ddot{y}_{\mathrm{u}}\end{array}\right\}, \quad$ and $R=\left\{\begin{array}{l}-m_{\mathrm{s}} \ddot{\xi} \\ -m_{\mathrm{u}} \ddot{\xi}\end{array}\right\}$

Solving Eqn. (2) for a given road profile produces the absolute displacements of the sprung and un-sprung masses such that

$$
y_{\mathrm{s}}^{\mathrm{t}}=y_{\mathrm{s}}+y_{\mathrm{u}}+\xi \quad \text { and } \quad y_{\mathrm{u}}^{\mathrm{t}}=y_{\mathrm{u}}+\xi
$$

\subsection{Roughness Generation}

The inverse fast Fourier transform (IFFT) of the power spectrum density (PSD) of a road profile ( $\xi$ ) provides a suitable model to represent profiles of different roughness levels (Wu 2000, Jiang, Cheng et al. 2012). The ISO (1995) (Andren 2006) represents the elevation profile as a PSD $S_{\xi}(\kappa)$ in the form

$$
S_{\xi}(\kappa)=\left\{\begin{array}{l}
S_{\xi}\left(\kappa_{0}\right)\left(\frac{\kappa}{\kappa_{0}}\right)^{-n_{1}} \frac{\kappa}{\kappa_{0}} \leq 1 \\
S_{\xi}\left(\kappa_{0}\right)\left(\frac{\kappa}{\kappa_{0}}\right)^{-n_{2}} \frac{\kappa}{\kappa_{0}}>1
\end{array}\right.
$$

where $\kappa$ is the wavenumber in units of cycle $/ \mathrm{m}, \kappa_{0}$ is the datum wavenumber in cycle $/ \mathrm{m}, S_{\xi}(\kappa)$ is in units of $\mathrm{m}^{3} /$ cycle, and $S_{\xi}\left(\kappa_{0}\right)$ is the PSD at $\kappa_{0}$ or initial PSD in $\mathrm{m}^{3} /$ cycle.

For typical profiles, Cebon (Cebon 1999) recommended $n_{1}=3, n_{2}=2.25$, and $\kappa_{0}=1 / 2 \pi$ cycles $/ \mathrm{m}$. Table 1 summarizes the qualitative relationship between the roughness classification and the initial PSD. That is, higher values of $S_{\xi}\left(\kappa_{0}\right)$ correspond to rougher roads. 
Table 1. Relationship between roughness classification and initial PSD (Cebon 1999)

\begin{tabular}{cc}
\hline Roughness classification & $S_{\xi}\left(\kappa_{0}\right), 10^{-6} \mathrm{~m}^{3} /$ cycle \\
\hline Very good & $2-8$ \\
Good & $8-32$ \\
Average & $32-128$ \\
Poor & $128-512$ \\
Very poor & $512-2048$ \\
\hline
\end{tabular}

Reference (Sayers and Karamihas 1998) indicates that the IRI quarter car model does not respond to spatial wavelengths that fall outside $1.3 \mathrm{~m}$ to $30 \mathrm{~m}$. Therefore, a range of $\kappa$ from $0.02 \mathrm{cycle} / \mathrm{m}$ to 5 cycle/m will conservatively excite the IRI quarter car model. Figure 2 shows the 1000 -meter road profile generated at $S_{\xi}\left(\kappa_{0}\right)=3.2 \times 10^{-5} \mathrm{~m}^{3} /$ cycle.

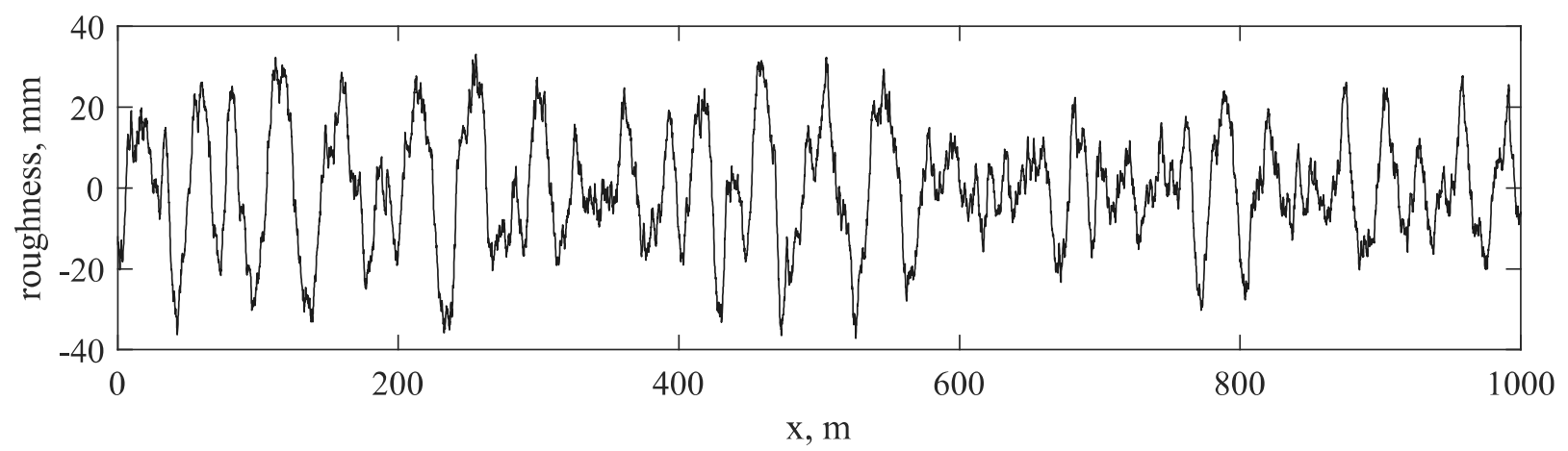

Figure 2. Generated road profile by IFFT method at $S_{\xi}\left(\kappa_{0}\right)=3.2 \times 10^{-5} \mathrm{~m}^{3} /$ cycle

\section{Wavelet decomposition of vehicle responses}

\subsection{Theory of wavelet analysis}

Researchers widely use the Fourier transform (FT) to characterize road profile as the sum of an infinite series of harmonic functions, namely the Fourier expansion (Sayers, Gillespie et al. 1986, Hayhoe 1992). A fundamental disadvantage of the Fourier expansion is that it represents the road profile only in the frequency domain and loses all the information in the spatial or time domain. Alternatively, using the 
Fourier spectra determines the frequency composition of a profile but it cannot detect the location of specific spatial characteristics such as the localized distress or defects.

In comparison, the wavelet transform (WT) represents a signal in the frequency and spatial/temporal domains simultaneously. It has the potential to characterize local features of the road elevation profile in both domains. Wavelet analysis moves stretched or dilated versions of a wavelet function across a signal to produce a correlation with local signal shapes that resemble the selected wavelet function. The wavelet $\psi(t)$ is a function of time $t$ corresponding to a variable resolution $a$ and location $b$ such that (Coifman, Meyer et al. 1992):

$$
\psi_{a, b}(t)=\frac{1}{\sqrt{a}} \psi\left(\frac{t-b}{a}\right)
$$

The scale factor $a$ is dimensionless and inversely proportional to the frequency of the selected wavelet, i.e., $f$, as (Addison 2017):

$$
f=\frac{f_{\mathrm{c}}}{a}
$$

where $f_{\mathrm{c}}$ is a constant of the selected wavelet.

The continuous wavelet transform (CWT) converts the signal $s(t)$ with a selected wavelet function $\psi_{a, b}(t)$ in an integral form such that (Coifman, Meyer et al. 1992):

$$
W_{a, b}=\int_{-\infty}^{+\infty} s(t) \psi_{a, b}(t) \mathrm{d} t
$$

where $W_{a, b}$ is the wavelet coefficient corresponding to the scale $a$ and the position $b$. The CWT traverses the scale $a$ continuously and slides the wavelet function across the signal. 
In comparison, the discrete wavelet transform (DWT) operates only for a certain range of frequency bands and selected signal locations. Hence, a representation of the time signal $s(t)$ is a series of sub-band signals $d_{m}(t)$ summed together such that (Coifman, Meyer et al. 1992):

$$
s(t)=\sum_{m=0}^{n} d_{m}(t)
$$

where $d_{m}$ stands for a signal within a certain frequency range $m$ that is preselected. The variation of $m$ shifts across the frequency bands that is of interest from 0 to $n$. Essentially, the wavelet transform processes the road profile series repeatedly at various resolutions to transform the road profile into different frequency bands. The design of the wavelet analysis procedure is such that it identifies both the features at the large and small scales.

\subsection{Wavelet decomposition of vehicle responses}

Sensitivity analysis in the authors' previous study (Zhang, Sun et al. 2018) indicates that the relationship between the vehicle responses, that is the sprung mass acceleration $a_{\mathrm{s}}$ the un-sprung mass acceleration $a_{\mathrm{u}}$ and the vehicle speed $v$ can be expressed with an exponential function wherein the power index denotes the contribution from the vehicle speed. Therefore, the present study minimizes the influence of the vehicle speed by dividing the acceleration to the average of the regressed powers, that is 0.7359 and 0.7669 for $a_{\mathrm{s}}$ and $a_{\mathrm{u}}$, respectively, which generates the normalized vehicle accelerations, $a_{\mathrm{s}}^{0}$ and $a_{\mathrm{u}}^{0}$ as shown in Eqns. (10) and (11). 


$$
a_{\mathrm{s}}^{0}=\frac{a_{\mathrm{s}}}{v^{0.7359}}
$$

$$
a_{\mathrm{u}}^{0}=\frac{a_{\mathrm{u}}}{v^{0.7669}}
$$

The MATLAB Wavelet Toolbox (Misiti, Misiti et al. 2004) is used to apply wavelet decomposition to both the road profile and the vehicle responses. Application of the WT requires using the vehicle speed to transform the road roughness data collected in the spatial domain to the temporal domain. The authors select the wavelet function DB3 from the Daubechies' wavelet family because it is the most widely used wavelet function to analyze road roughness and vehicle dynamic responses. Figure 3 plots the shape of the DB3 wavelet function.

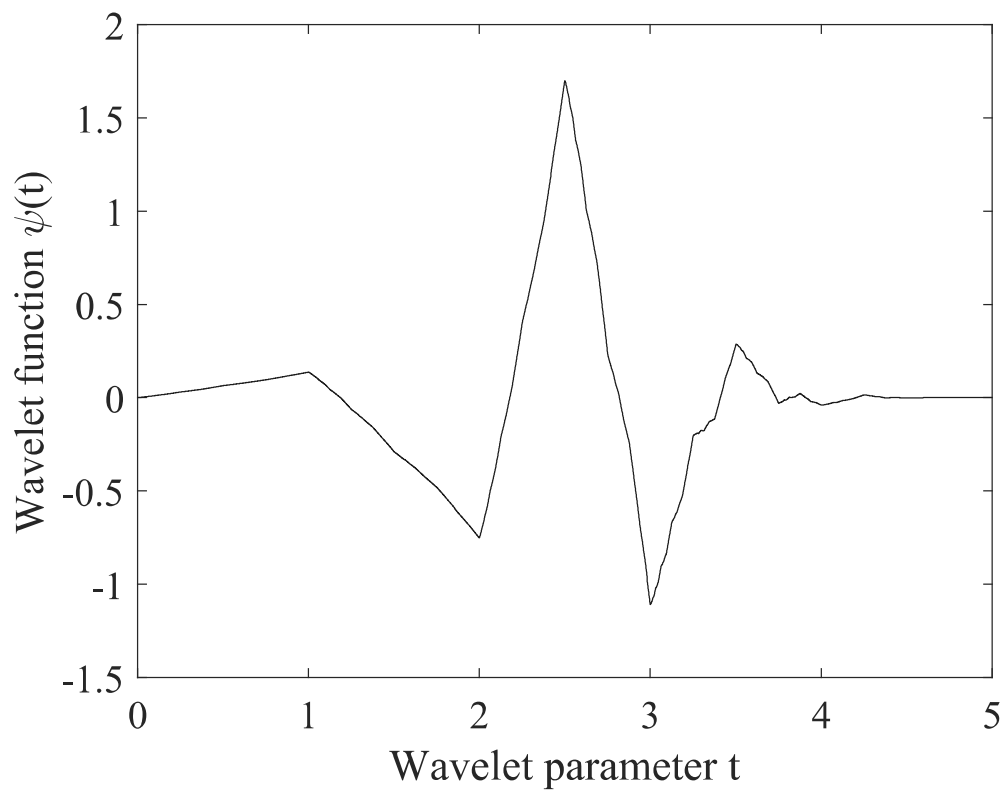

Figure 3. Daubechies wavelet function DB3

The discrete wavelet transform (DWT) produces a sparse representation of the natural signals avoiding the redundancy in the continuous wavelet transform (CWT). In DWT, the original signal $s(t)$ represents the measured road roughness data or vehicle inertial responses, and $t$ now denotes time. A mathematical expression of $s(t)$ is the sum of the wavelet decomposed components such that: 


$$
s(t)=d_{1}(t)+d_{2}(t)+\ldots+d_{n}(t)+a_{n}(t), \quad \text { subject to } 2^{n} \leq N
$$

where $d_{i}(t)$, for $i=0,1,2, \ldots, n$ is the sub-band signal corresponding to the high frequency sub-bands selected (detail), $a_{n}(t)$ corresponds to the low frequency sub-band at the $n^{\text {th }}$ level (approximation), and $n$ is the highest level of wavelet decomposition that is subject to the condition that $2^{n} \leq N$ where $N$ is total number of data points (Addison 2017). A trial analysis in this present study indicates that a five-level wavelet decomposition provides sufficient details for the road roughness and vehicle inertial response analysis. At a sampling rate of $222 \mathrm{~Hz}$, the frequency ranges of $d_{1}, d_{2}, d_{3}, d_{4}, d_{5}$ and $a_{5}$ are 56-111, 28$56,14-28,7-14,3-7$, and $0-3 \mathrm{~Hz}$, respectively. The frequency ranges except that of $a_{5}$ have a upper limit equal to two times the lower frequency limit, which is the result of wavelet decomposition using the dyadic scales and positions (Misiti, Misiti et al. 2004, Addison 2017). Figure 4 illustrates the structure of the fivelevel wavelet decomposition.

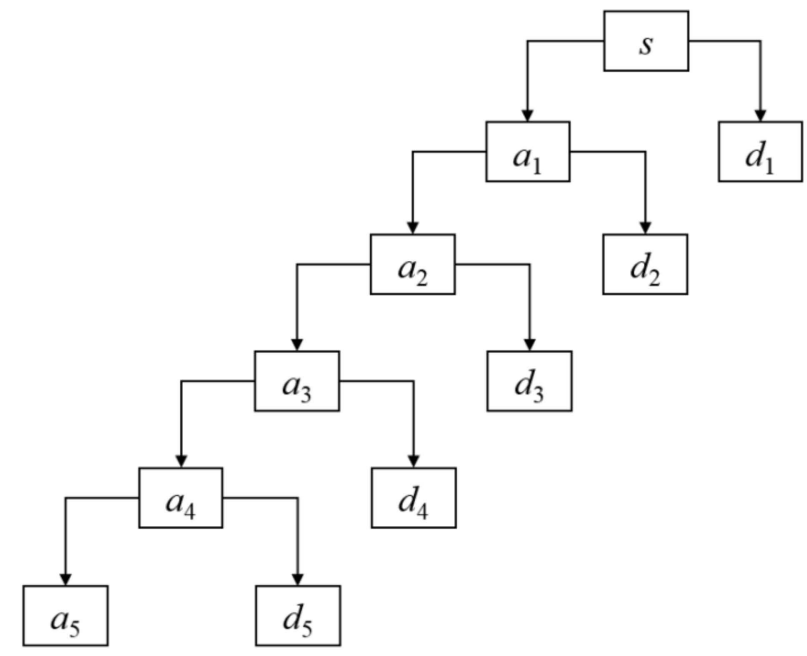

Figure 4. Five-level wavelet decomposition

The DB3 wavelet function decomposed to the fifth level the road profile generated at $S_{\xi}\left(\kappa_{0}\right)=3.2 \times 10^{-5} \mathrm{~m}^{3} /$ cycle. Figure 5 illustrates the original profile $(s)$ and the decomposed components $\left(d_{1}-d_{5}\right.$ and $\left.a_{5}\right)$. Most of the roughness signal's energy $(96.7 \%)$ is concentrated in the approximation component $a_{5}$ which resembles the original profile $s$ after denoising. Each of the high frequency components $\left(d_{1}-d_{5}\right)$ occupy much less energy than $a_{5}$. Figure 6 shows the wavelet decomposition of the normalized 
acceleration of a quarter car's sprung mass $a_{\mathrm{s}}^{0}$ under the excitation of the roughness profile generated at $S_{\xi}\left(\kappa_{0}\right)=3.2 \times 10^{-5} \mathrm{~m}^{3} /$ cycle. The travelling speed is $80 \mathrm{~km} / \mathrm{h}$. Differing from the frequency components of the roughness signal, a considerable portion of energy lies in the $4^{\text {th }}$ and $5^{\text {th }}$ level details of the sprung mass acceleration. Specifically, $26.3 \%$ and $14.3 \%$ of the energy is contained in $d_{4}$ and $d_{5}$, respectively. The level in $a_{5}$ contains most (50.8\%) of the signal's energy. Section 4 will use the frequency components of the sprung mass acceleration from the wavelet analysis and their combinations for road profile reconstruction.

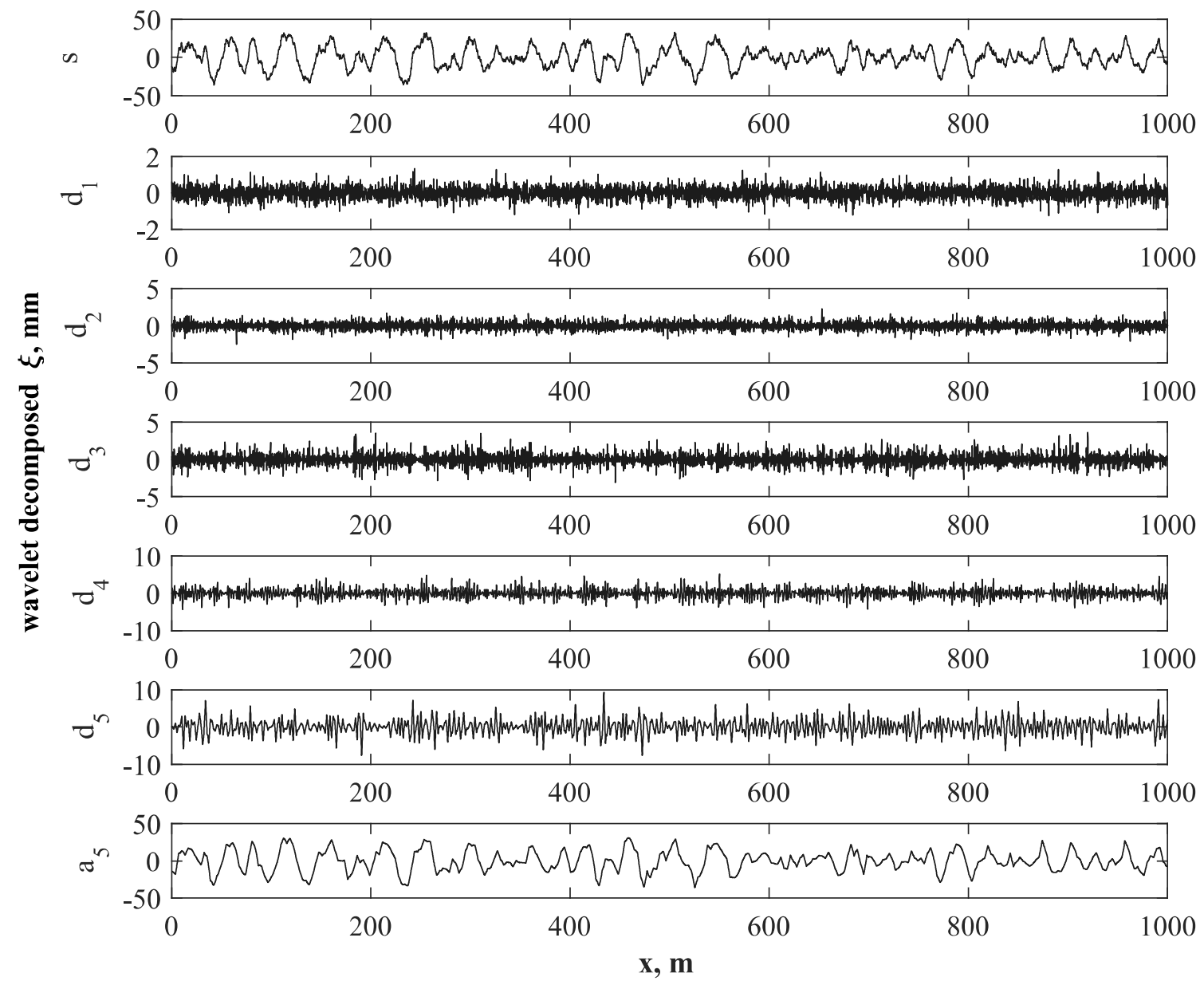

Figure 5. Five-level wavelet analysis of roughness generated at $S_{\xi}\left(\kappa_{0}\right)=3.2 \times 10^{-5} \mathrm{~m}^{3} /$ cycle 


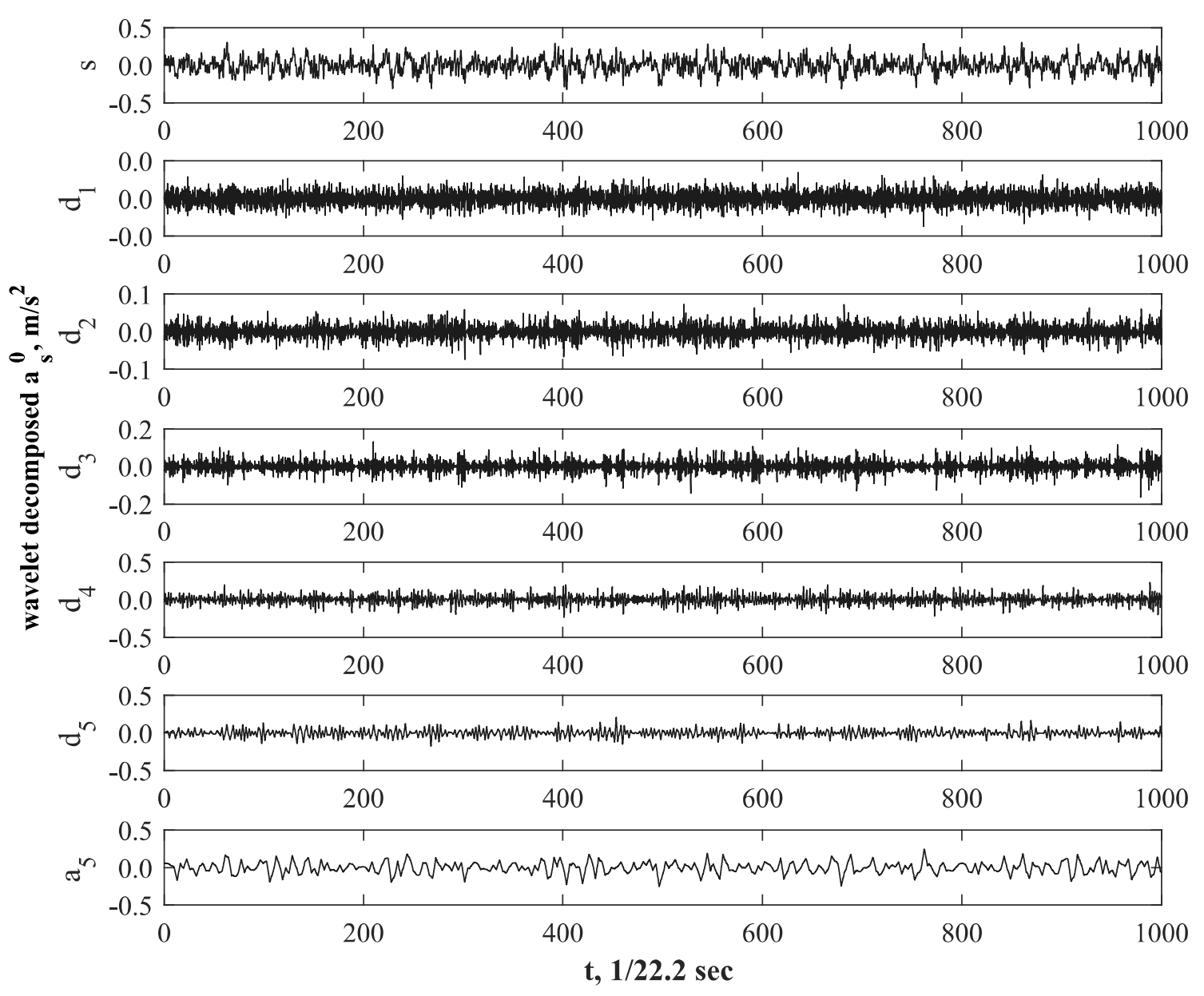

Figure 6. Five-level wavelet analysis of $a_{\mathrm{s}}^{0}$ at $S_{\xi}\left(\kappa_{0}\right)=3.2 \times 10^{-5} \mathrm{~m}^{3} /$ cycle

\section{Road Profile Reconstruction}

\subsection{NARX neural network}

Artificial neural networks (ANN) consist of simple elements (neurons) that operate in parallel. Researchers widely use the ANN to model nonlinear dynamic system due to their adaptability and ready availability (Wong and Worden 2007). In this paper, the authors describe the selection and training of one type of ANN to identify the inertial response of a vehicle under the excitation of road surface roughness, and subsequently to reconstruct the road surface profile. The profile inputs and the vehicle inertial responses are linked in a nonlinear manner (Ngwangwa, Heyns et al. 2010). 
The nonlinear autoregressive network with exogenous inputs (NARX) is a type of ANN that is popular in nonlinear dynamic model simulations (Wong and Worden 2007). The NARX network is a recurrent dynamic network that has feedback connections enclosing several layers. Wong and Worden (Wong and Worden 2007) indicated that a three-layer multilayer perceptron (MLP) NARX is sufficient to perform an input-output mapping for any continuous function. The MLP network takes the system input $\mathbf{u}(t-i)$ and the past output values $\xi_{\mathrm{r}}(t-i)$ as the input for computing the current output values. The operating principle of a NARX network is expressed (Demuth and Beale 2009) as:

$$
\tilde{\xi}_{\mathrm{r}}(t)=\mathbf{g}\left(\mathbf{u}\left(t-n_{\mathrm{u}}\right), \ldots, \mathbf{u}(t-1), \mathbf{u}(t), \xi_{\mathrm{r}}\left(t-n_{\mathrm{z}}\right), \ldots, \xi_{\mathrm{r}}(t-1)\right)
$$

The function $\mathbf{g}()$ is the nonlinear function of the input-output relationship MLP mapping. The function $\mathbf{u}(t)$ is a vector containing the network input sequences that can be the vehicle sprung mass acceleration or its wavelet decomposed components. The function $\xi_{\mathrm{r}}(t)$ is the network target, which this analysis specifies as the profile elevation at time $t$. The parameters $n_{\mathrm{u}}$ and $n_{\mathrm{z}}$ are the maximum delays of input and output, respectively.

Figure 7 illustrates the proposed framework using the NARX network to reconstruct the road elevation profile. The present study uses the adapted series-parallel architecture to reduce the computational cost. The series-parallel architecture does not have the feedback loop that increases the computation efforts and applies a static backpropagation method to adjust the model weight parameters. Training and implementation of the network assumes a stable vehicle-road system with bounded inputs and outputs within the variable model parameters. A Levenberg-Marquardt algorithm trains the initiated network by updating the model weights as follows (Demuth and Beale 2009): 


$$
\mathbf{w}_{\text {new }}=\mathbf{w}_{\text {old }}+\left(\mathbf{J}^{\mathrm{T}} \mathbf{J}+\lambda \mathbf{I}\right)^{-1} \mathbf{J}^{\mathrm{T}} \boldsymbol{\varepsilon}\left(\mathbf{w}_{\text {old }}\right)
$$

where $\mathbf{w}_{\text {new }}$ is the vector of new weights in the next round, $\mathbf{w}_{\text {old }}$ is the vector of current weights, $\mathbf{I}$ is the identity matrix, $\boldsymbol{\varepsilon}\left(\mathbf{w}_{\text {old }}\right)$ is an error vector of the current point, $\mathbf{J}$ is a Jacobian matrix consisting of the derivatives of the error vector $\boldsymbol{\varepsilon}\left(\mathbf{w}_{\text {old }}\right)$ with respect to the parameters, and $\lambda$ is a parameter governing the step size of iteration. The method minimizes the error vector $\boldsymbol{\varepsilon}\left(\mathbf{w}_{\text {old }}\right)$ during the network training. The authors select the Levenberg-Marquardt algorithm for the network training due to its efficiency in function approximation and high computational efficiency by avoiding the evaluation of the Hessian matrix that is much more computationally expensive (Demuth and Beale 2009).

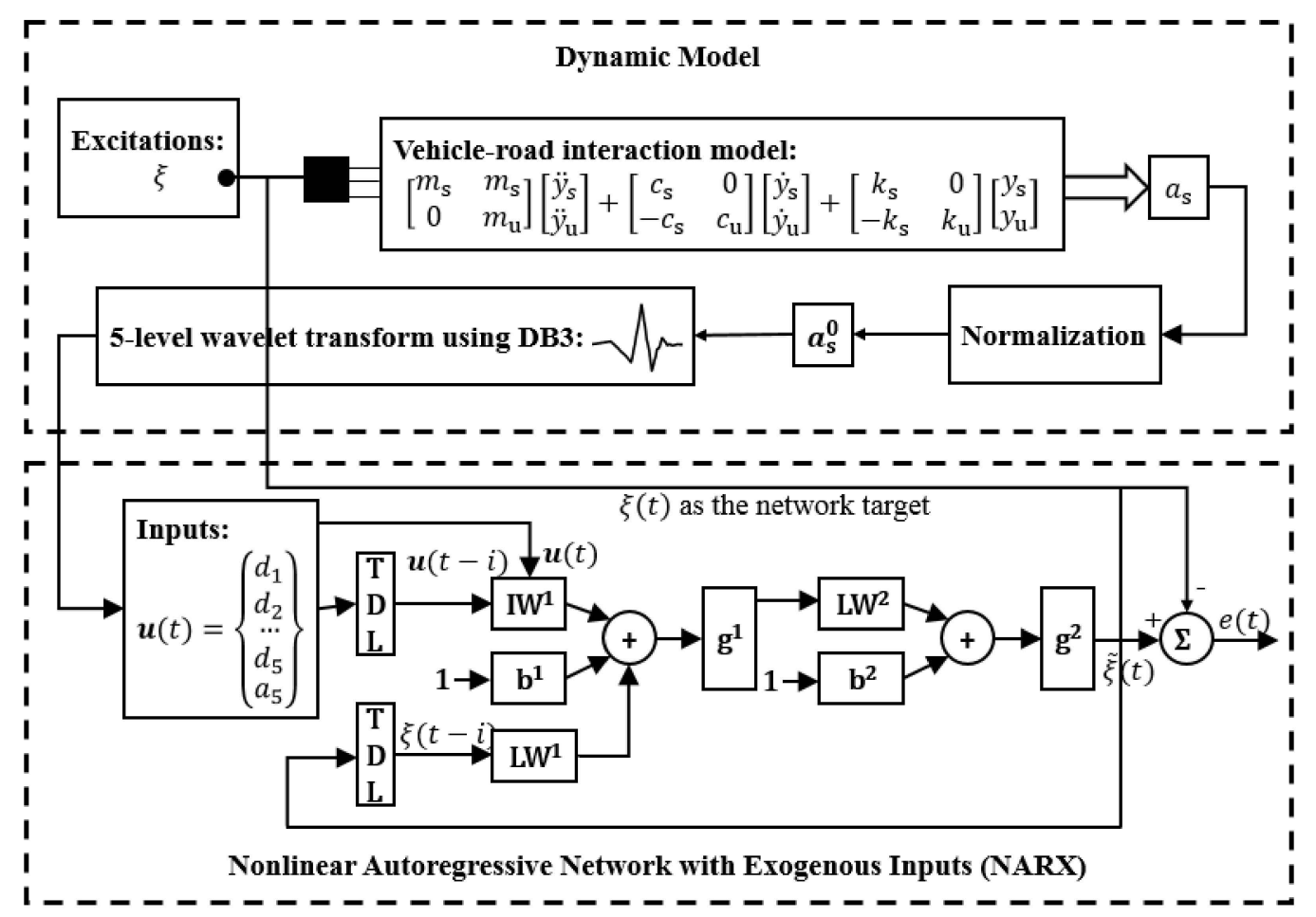

Figure 7. A series-parallel architecture of the NARX model used for road surface profile reconstruction

\subsection{Road Profile Reconstruction}

This subsection demonstrates the proposed methodology introduced in Section 4.1 for road profile reconstruction at different roughness levels. Figure 8 illustrates the architecture of the NARX network used for road profile reconstruction where $x(t), y(t)$ denote the input and the prediction. The figure shows that 
the network has a hidden layer of 10 neurons and an output layer composed of one neuron. The optimization with a large number of trial analysis determined that the number of delays should be four.

The network used applies the embedded training function trainbr in MATLAB to update the model parameters using the Levenberg-Marquardt algorithm described via Eqn. (14). The Levenberg-Marquardt algorithm optimizes the generalization of the network by minimizing the combined squared errors and weights of the model (Bishop 1995, Demuth and Beale 2009).

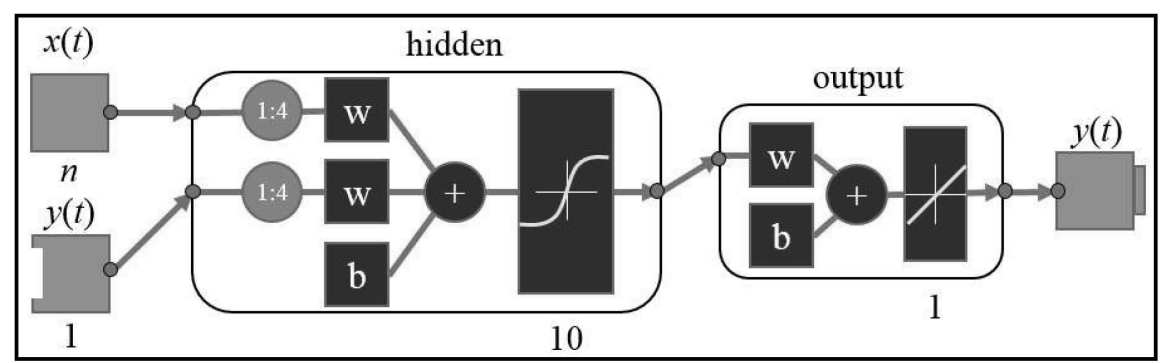

Figure 8. The architecture of the NARX network for road profile reconstruction. Modified based on (Demuth and Beale 2009).

The road section used in this study has a length of $1000 \mathrm{~m}$. The authors generated one hundred road roughness profiles for each of the roughness categories listed in Table 1, and simulated the quarter car model to obtain the corresponding IRI values. These profiles, 600 in total, are inputs to the NARX network for training, validation, and testing with a 10 -fold cross validation.

Figure 9(a) illustrates the results of profile reconstruction using the sprung mass acceleration $a_{\mathrm{s}}^{0}$ from profile roughness generated at $S_{\xi}\left(\kappa_{0}\right)=3.2 \times 10^{-5} \mathrm{~m}^{3} /$ cycle. Figure $9(\mathrm{~b})$ shows more details in an amplified section of the plot. The plot "target" is the generated roughness value at a specific location, and "output" is the reconstructed roughness using the NARX network. A comparison between the "target" and the "output" indicates a high quality of road profile reconstruction using $a_{\mathrm{s}}^{0}$ as the input. However, the amplified plot of Figure 9(b) illustrates deviations in the local fluctuations. This result indicates that road profile reconstruction using $a_{\mathrm{s}}^{0}$ as the input is limited because the reproduction lacks local details that may indicate defects such as rutting, cracking, and potholes. 


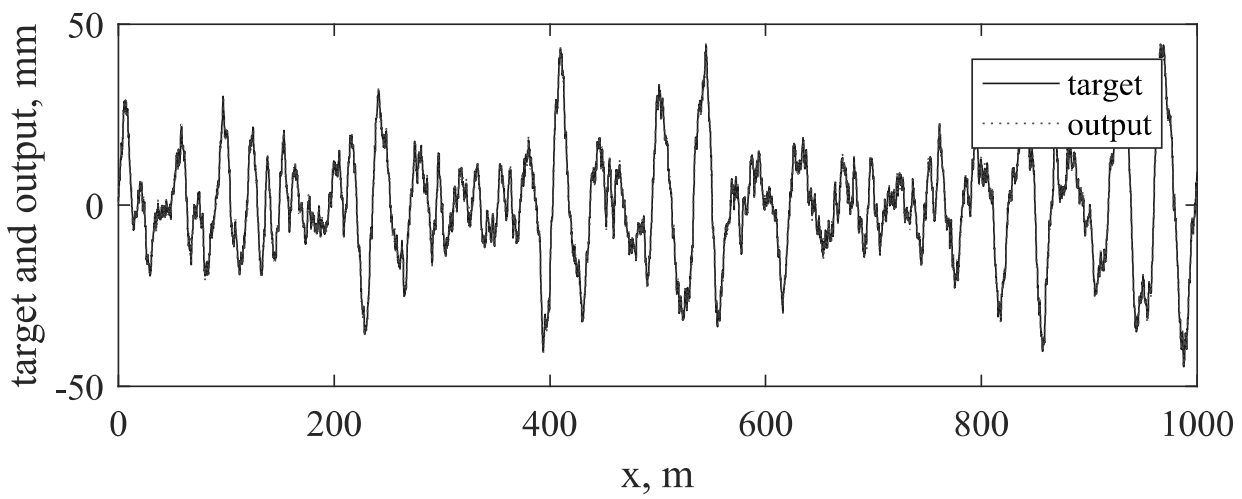

(a) Comparison between the target and the reconstructed road profile

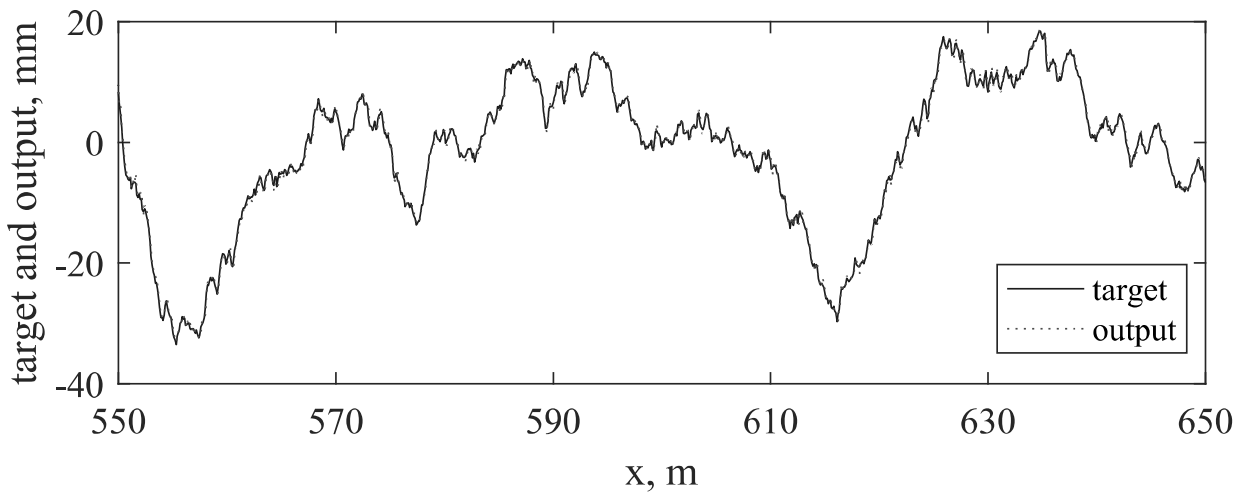

(b) 100-meter amplification

Figure 9. Results of profile reconstruction using $s\left(a_{\mathrm{s}}^{0}\right)$ at $S_{\xi}\left(\kappa_{0}\right)=3.2 \times 10^{-5} \mathrm{~m}^{3} /$ cycle

Figure 10(a) and 10(b) show the profile reconstruction using the six signal components $d_{1} \sim d_{5}$ and $a_{5}$ obtained from the five-level wavelet analysis. In the training process, the NARX network selects and combines features from the six wavelet signals of the different frequency bands that contribute the most towards minimizing the error at each location of the profile reconstruction. As a result, the reconstructed profile using $d_{1} \sim d_{5}$ and $a_{5}$ not only recovers well the overall trend of the target profile but captures the local details better than that of the reconstruction using only $a_{\mathrm{s}}^{0}$. A contrast of the output shown in Figure 10(b) with that shown in Figure 9(b) indicates a better agreement of the positions and amplitudes of the local curvatures with the target profile. Figure 11(a) and (b) illustrate the distribution of the profile reconstruction errors using $a_{\mathrm{s}}^{0}$ and its wavelet decomposition components $d_{1} \sim d_{5}$ and $a_{5}$, respectively. Both error series apparently follow normal distributions as expected. The mean value is close to zero 
$\left(7.98 \times 10^{-5} \mathrm{~mm}\right.$ and $-7.83 \times 10^{-4} \mathrm{~mm}$, respectively). Table 2 summarizes their statistics. A $26 \%$ smaller value of root mean square quantifies the smaller deviation of the reconstructed profile from zero error, and thus denotes a better quality of profile reconstruction using the combined wavelet decomposition components $d_{1} \sim d_{5}$ and $a_{5}$ than using $a_{\mathrm{s}}^{0}$.

Using step functions to represent sharp road bumps serves as another illustration of the reconstruction effectiveness. Figure 12 (a) shows the reconstruction quality using only $a_{\mathrm{s}}^{0}$ whereas Figure 12 (b) shows the reconstruction quality using $d_{1} \sim d_{5}$ and $a_{5}$. Apparently, the latter results diverge less from the target profile. In summary, the application of an ANN to a wavelet decomposition of the vehicle inertial responses will further improve the reconstruction quality of the elevation profile in representing local road surface defects.

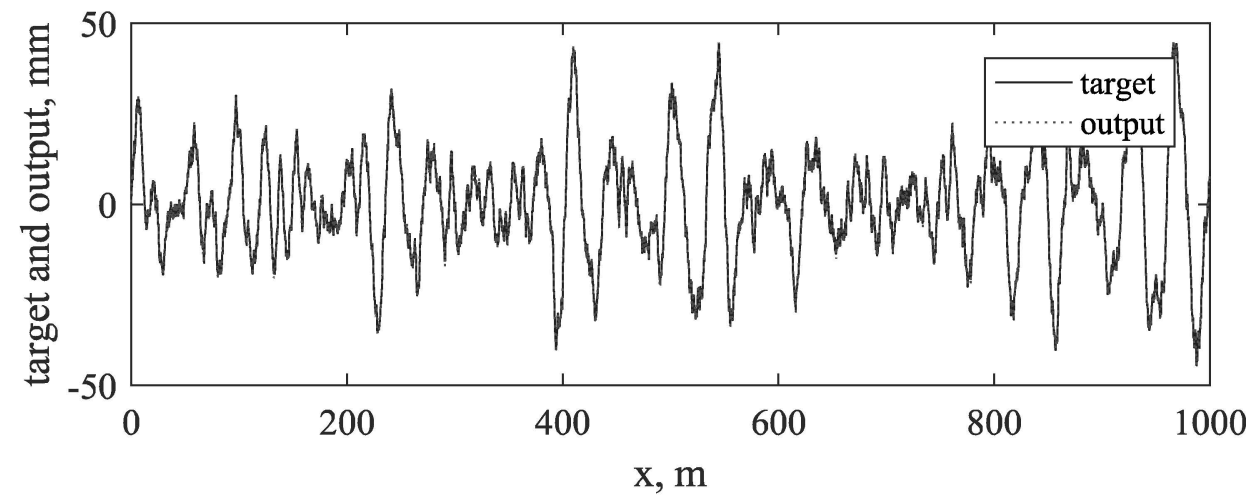

(a) full profile

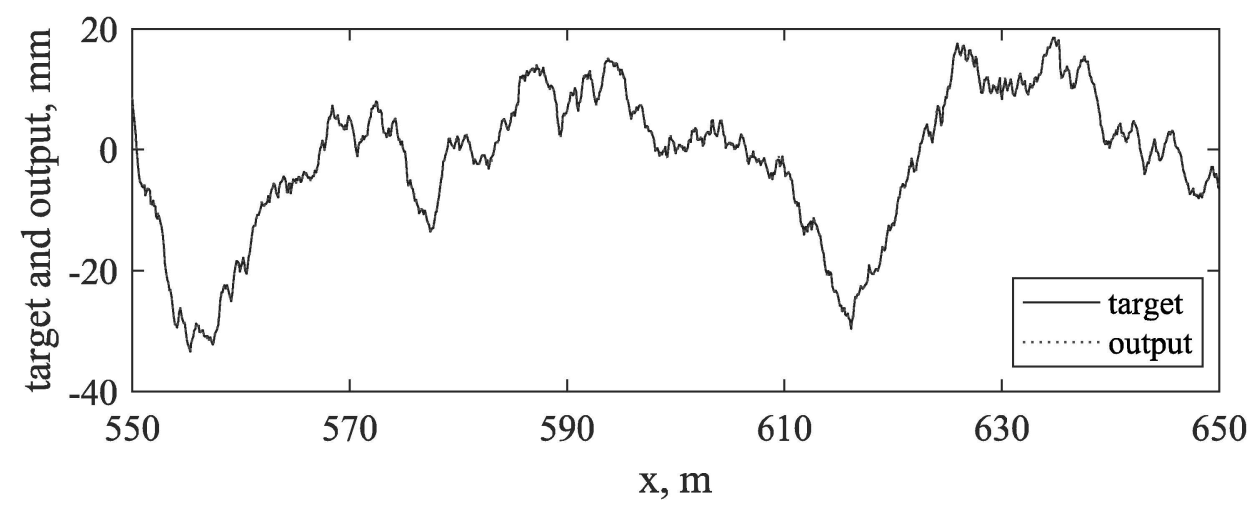

(b) 100-meter amplification

Figure 10. Results of profile reconstruction using $d_{1} \sim d_{5}$ and $a_{5}$ at $S_{\xi}\left(\kappa_{0}\right)=3.2 \times 10^{-5} \mathrm{~m}^{3} / \mathrm{cycle}$ 


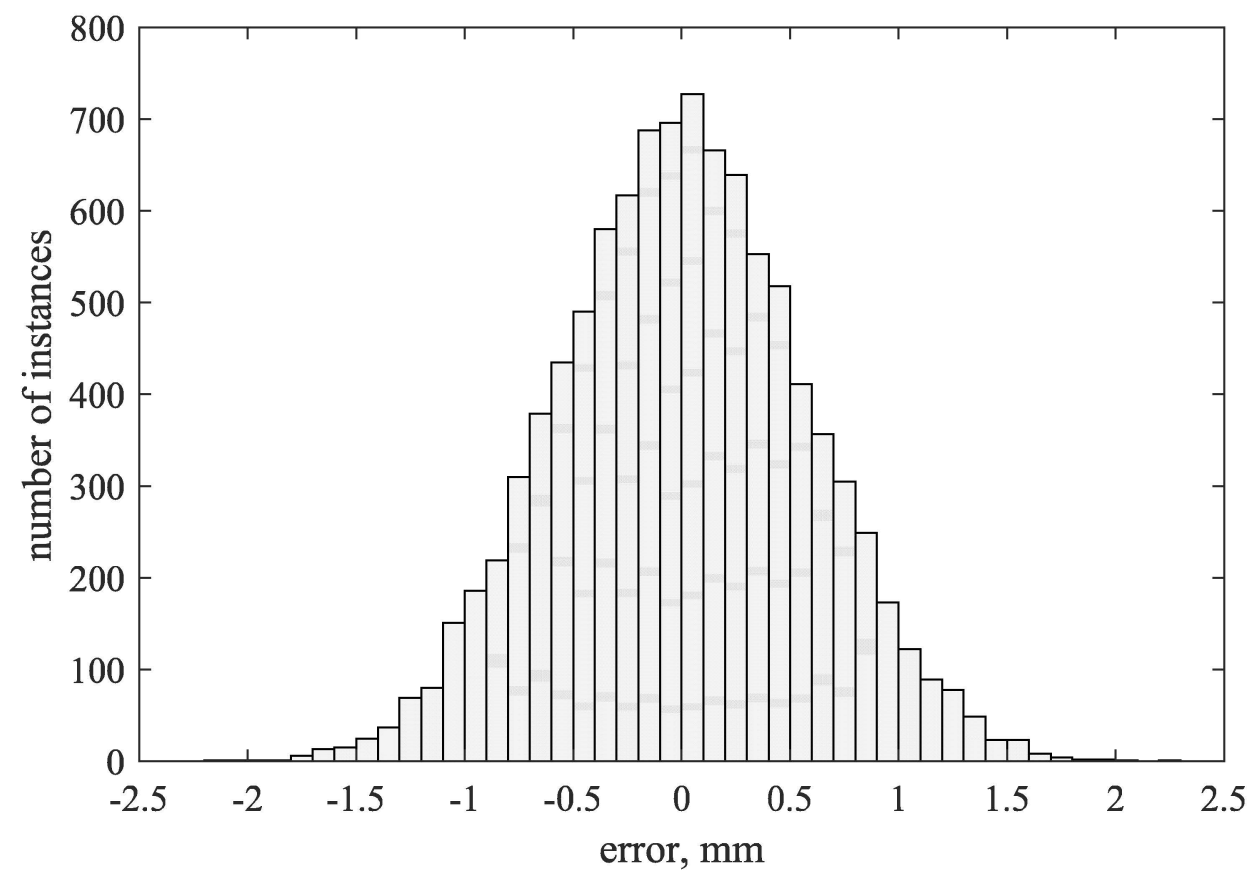

(a) $a_{\mathrm{s}}^{0}$

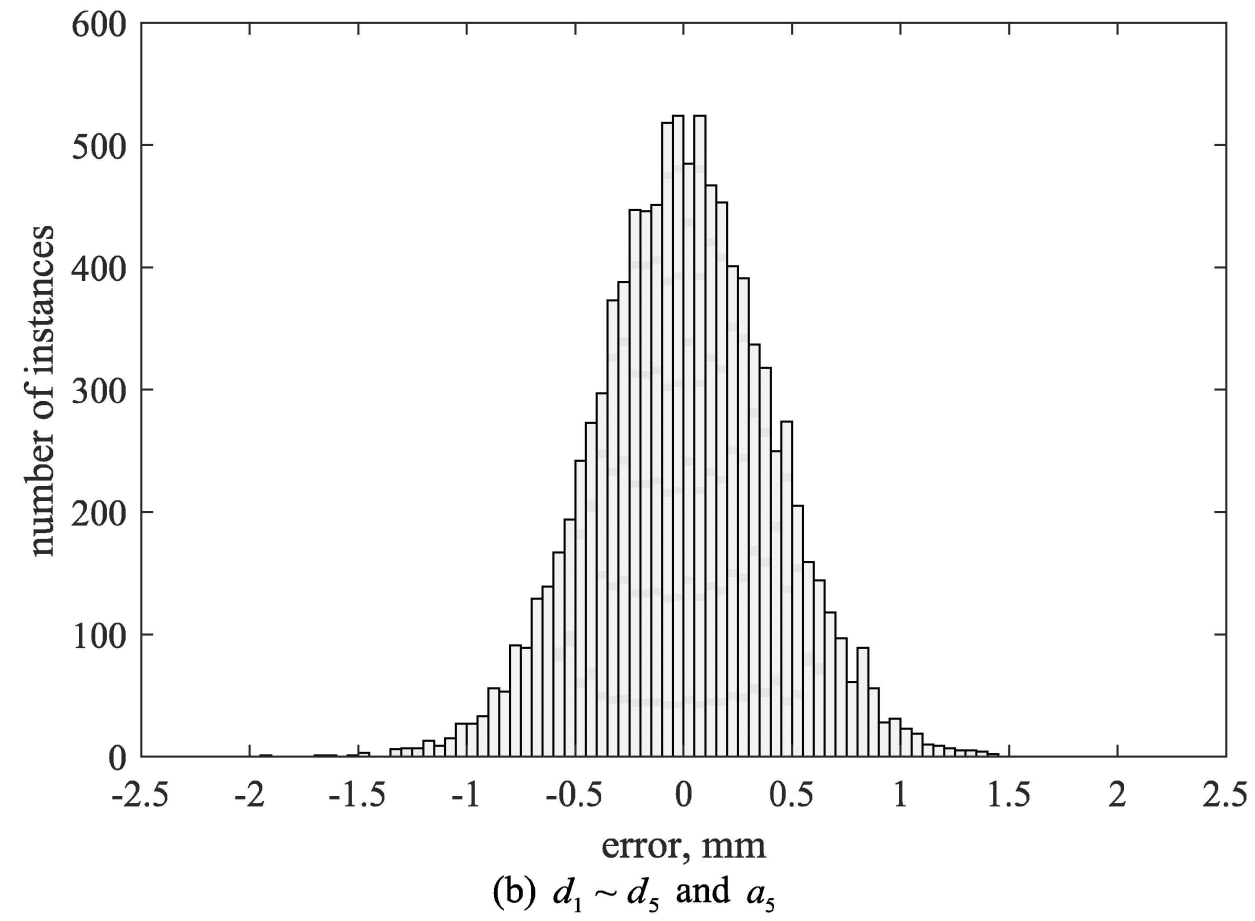

Figure 11. Profile reconstruction error distribution at $S_{\xi}\left(\kappa_{0}\right)=3.2 \times 10^{-5} \mathrm{~m}^{3} / \mathrm{cycle}$

Table 2. Statistics of profile reconstruction errors at $S_{\xi}\left(\kappa_{0}\right)=3.2 \times 10^{-5} \mathrm{~m}^{3} / \mathrm{cycle}$ 


\begin{tabular}{cccc}
\hline$a_{\mathrm{s}}^{0}$ & $7.98 \times 10^{-5}$ & 0.57 & 0.55 \\
\hline$d_{1} \sim d_{5}$ and $a_{5}$ & $-7.83 \times 10^{-4}$ & 0.40 & 0.41 \\
\hline
\end{tabular}
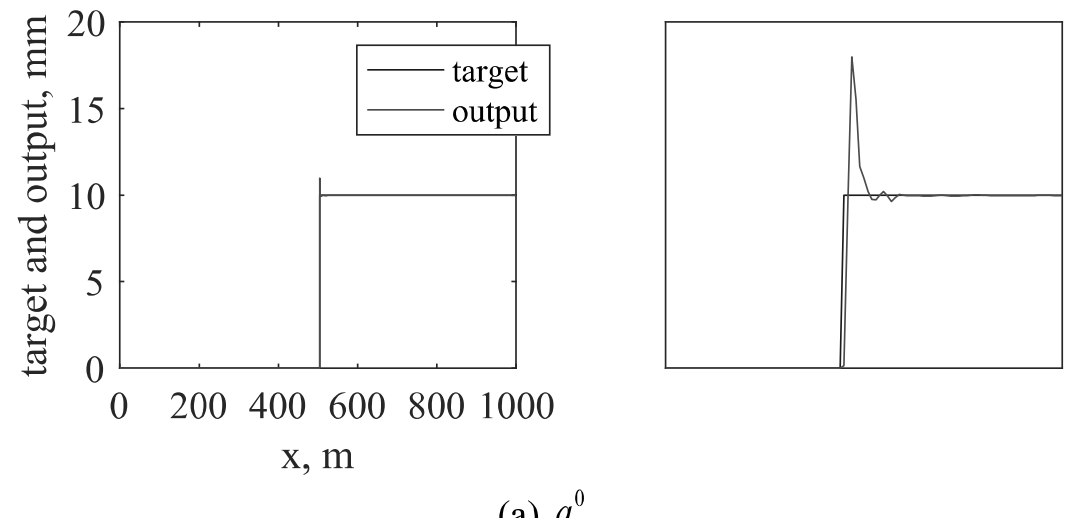

(a) $a_{\mathrm{s}}^{0}$
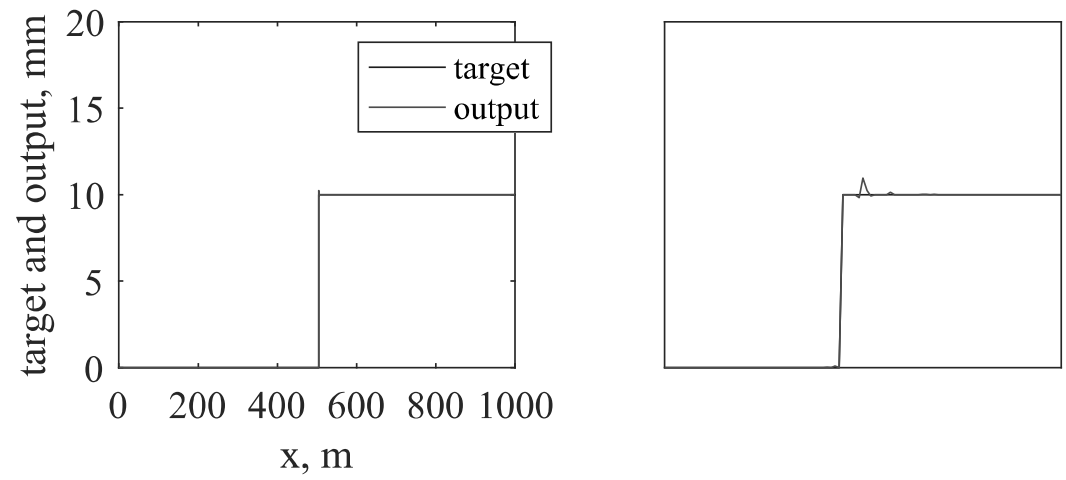

(b) $d_{1} \sim d_{5}$ and $a_{5}$

Figure 12. Profile reconstruction of a step function. (a) use $a_{\mathrm{s}}^{0}$ as input; (b) use wavelet analysis components $d_{1} \sim d_{5}$ and $a_{5}$ as input.

As shown in Figure 13, the authors developed a metric to compare the results of road profile reconstruction using different inputs. Table 3 lists the roughness levels labeled on the left side of the figure. The "max error" denotes the maximum profile difference divided by the peak value of the target profile; the SSE in percentage is the ratio of the sum of squares of errors (SSE) divided by the sum of squares of regression (SSR) plus SSE, that is SSE/(SSE+SSR); the IRI error is the relative difference of IRI.

Table 3. PSDs of roughness used for profile reconstruction

\begin{tabular}{ll}
\hline$S_{\xi}\left(\kappa_{0}\right)_{1}$ & $2 \times 10^{-6} \mathrm{~m}^{3} /$ cycle \\
\hline$S_{\xi}\left(\kappa_{0}\right)_{2}$ & $8 \times 10^{-6} \mathrm{~m}^{3} /$ cycle
\end{tabular}




\begin{tabular}{cc}
\hline$S_{\xi}\left(\kappa_{0}\right)_{3}$ & $3.2 \times 10^{-5} \mathrm{~m}^{3} / \mathrm{cycle}$ \\
\hline$S_{\xi}\left(\kappa_{0}\right)_{4}$ & $1.28 \times 10^{-4} \mathrm{~m}^{3} / \mathrm{cycle}$ \\
\hline$S_{\xi}\left(\kappa_{0}\right)_{5}$ & $5.12 \times 10^{-4} \mathrm{~m}^{3} / \mathrm{cycle}$ \\
\hline$S_{\xi}\left(\kappa_{0}\right)_{6}$ & $2.048 \times 10^{-3} \mathrm{~m}^{3} / \mathrm{cycle}$ \\
\hline
\end{tabular}

Figure 13 shows that for each of the roughness levels from $S_{\xi}\left(\kappa_{0}\right)_{1}$ to $S_{\xi}\left(\kappa_{0}\right)_{6}$ the profile reconstruction using the combined input of $d_{1} \sim d_{5}$ and $a_{5}$ provides the best performance because it has the lowest error evaluation indices (max error, SSE, and IRI error.) The second best performance is achieved by profile reconstruction using $s\left(a_{\mathrm{s}}^{0}\right)$, which performs better than that using any single wavelength signal component $\left(d_{1}, d_{2}, \ldots, d_{5}\right.$ or $a_{5}$.) Figure 13 further indicates that a single wavelet component of the vehicle response cannot represent well all the information about the road roughness contained within the response, and thus is insufficient for reconstructing the road profile. In addition, a comparison among the sub-figures in Figure 13 indicates that the quality of profile reconstruction does not vary significantly between the roughness levels.

Figure 14 evaluates the profile reconstruction quality using a comprehensive evaluation index, the integrated error (IE), which is derived by scaling the three evaluation indices shown in Figure 13 to the same scale based on their average values, that is then summed and normalized to the range [0 1]. The results agree with the previous evaluation in that the profile reconstruction, using $d_{1} \sim d_{5}$ and $a_{5}$, provides the best performance at all roughness levels. 

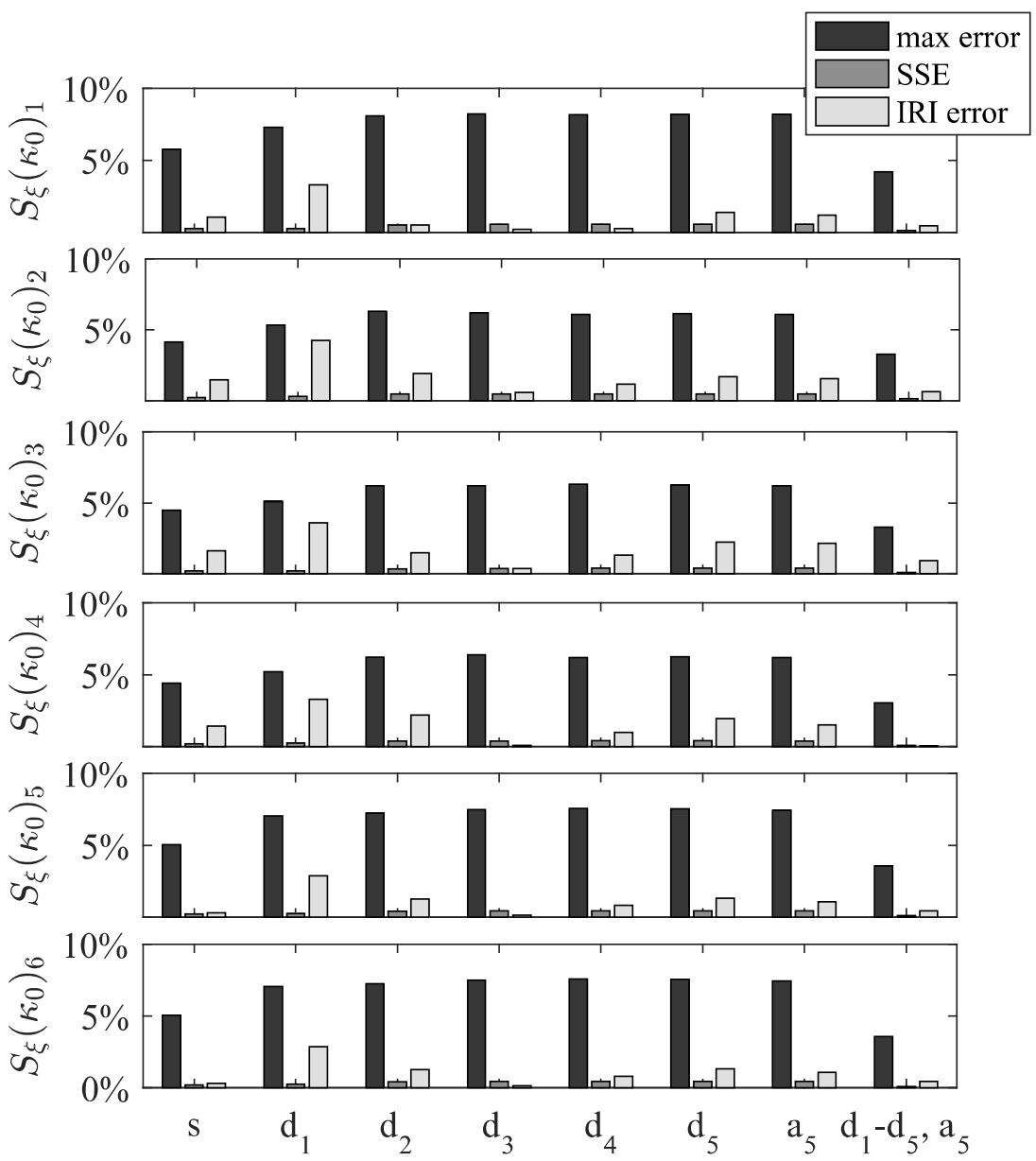

Figure 13. Evaluation of the profile reconstruction results using the NARX network

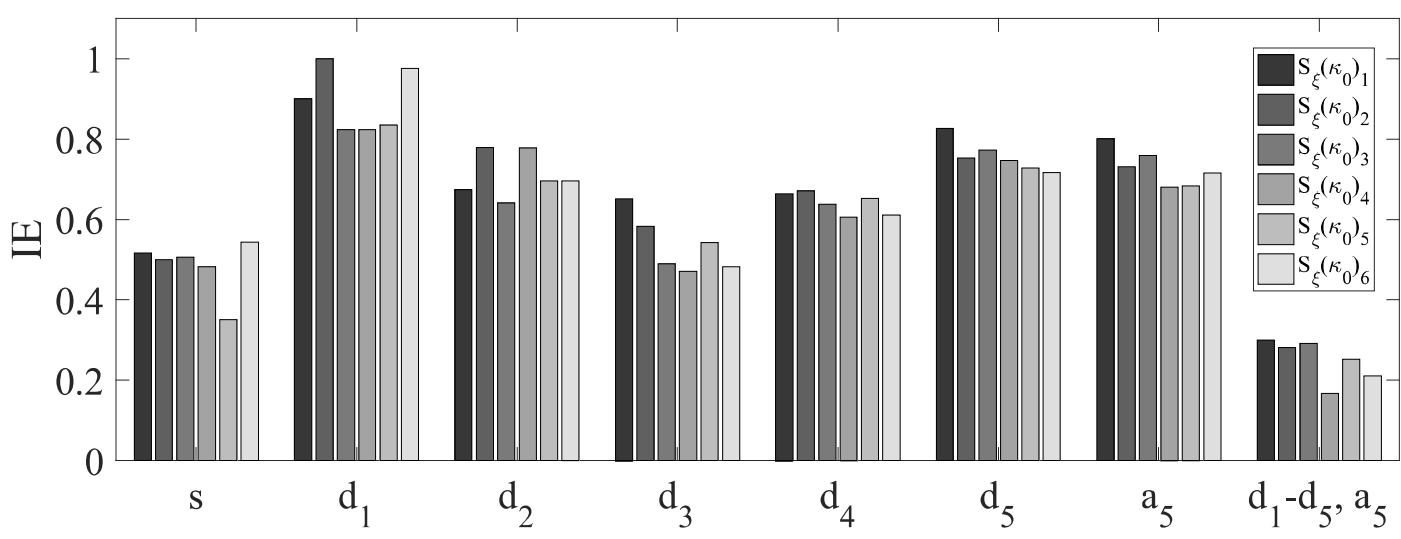

Figure 14. Comparison of integrated error (IE) using different signal components 


\section{Conclusions and Future Work}

The present study proposes a method of road profile reconstruction using wavelet decomposition of probe vehicle inertial responses and artificial neural network. The proposed method is implemented through numerical simulation using a quarter vehicle travelling across generated road section of different roughness levels from very good to very poor condition. The simulated vehicle response is normalized to the travelling speed using the results of sensitivity analysis and statistical regression. This study uses the wavelet function DB3 from the Daubechies family to decompose the road profile and vehicle acceleration to the fifth level, which shows that the signal energy concentration differs across the frequency bands. The selected neural network is a nonlinear autoregressive network with exogenous inputs (NARX) that captures the behavior of the nonlinear dynamic vehicle-road interaction system. The authors demonstrated the efficiency of the proposed methodology for various roughness levels. Comparison among the profiles reconstructed using different wavelet signal components indicates that the combined components from a wavelet decomposition of the vehicle responses best reproduces the roughness details of the original elevation profile, relative to using the dynamic inertial responses alone. A comprehensive and objective evaluation metric demonstrates the superiority of using this approach to reconstruct the road elevation profile from connected vehicle data.

Future work will include a parametric study on the influence from the vehicle properties, measurement accuracy, noise level, sampling rate and resolution. It will also include a practical implementation of the proposed methodology with field experiments across real road sections.

\section{Acknowledgment}

This work was supported by Louisiana Transportation Research Center [grant number is DOTLT1000138] and Louisiana State University Start-up Fund [grant number is 127150013]. The authors are grateful for all the support. 


\section{Reference}

Addison, P. S. (2017). The illustrated wavelet transform handbook: introductory theory and applications in science, engineering, medicine and finance, $\mathrm{CRC}$ press.

Andren, P. (2006). "Power spectral density approximations of longitudinal road profiles." International Journal of Vehicle Design 40(1): 2-14.

Attoh-Okine, N. O. (1999). "Analysis of learning rate and momentum term in backpropagation neural network algorithm trained to predict pavement performance." Advances in Engineering Software 30(4): 291-302.

Bishop, C. M. (1995). Neural networks for pattern recognition, Oxford university press.

Bridgelall, R. (2014). A participatory sensing approach to characterize ride quality. SPIE Smart Structures and Materials+ Nondestructive Evaluation and Health Monitoring, International Society for Optics and Photonics.

Bridgelall, R., Y. Huang, Z. Zhang and F. Deng (2016). "Precision enhancement of pavement roughness localization with connected vehicles." Measurement Science and Technology 27(2): 025012.

Cebon, D. (1999). Handbook of vehicle-road interaction.

Coifman, R. R., Y. Meyer and V. Wickerhauser (1992). Wavelet analysis and signal processing. In Wavelets and their applications, Citeseer.

Daubechies, I. (1988). "Orthonormal bases of compactly supported wavelets." Communications on pure and applied mathematics 41(7): 909-996.

De Pont, J. and A. Scott (1999). "Beyond road roughness--interpreting road profile data." Road \& Transport

Research 8(1): 12.

Demuth, H. and M. Beale (2009). "Matlab neural network toolbox user's guide version 6."

Guarneri, P., G. Rocca and M. Gobbi (2008). "A neural-network-based model for the dynamic simulation of the

tire/suspension system while traversing road irregularities." IEEE transactions on neural networks 19(9): 1549-1563.

Hayhoe, G. F. (1992). Spectral characteristics of longitudinal highway profiles as related to ride quality. Vehicle,

Tire, Pavement Interface, ASTM International.

Hoffmann, M., M. Mock and M. May (2013). Road-quality classification and bump detection with bicycle-mounted smartphones. Proceedings of the 3rd International Conference on Ubiquitous Data Mining-Volume 1088, CEUR-

WS. org.

Jiang, C. D., L. Cheng, S. Fengchun and C. Hongjie (2012). Simulation of Road Roughness Based on Using IFFT

Method. Software Engineering (WCSE), 2012 Third World Congress on, IEEE.

Kong, Y., S. Abdullah, D. Schramm, M. Omar, S. Haris and T. Bruckmann (2017). "Mission profiling of road data measurement for coil spring fatigue life." Measurement 107: 99-110.

Kong, Y., M. Z. Omar, L. Chua and S. Abdullah (2014). "Ride quality assessment of bus suspension system through modal frequency response approach." Advances in Mechanical Engineering 6: 269721.

Misiti, M., Y. Misiti, G. Oppenheim and J.-M. Poggi (2004). "Matlab Wavelet Toolbox Userl's Guide. Version 3." Ngwangwa, H. M., P. S. Heyns, F. Labuschagne and G. K. Kululanga (2010). "Reconstruction of road defects and road roughness classification using vehicle responses with artificial neural networks simulation." Journal of Terramechanics 47(2): 97-111.

Papagiannakis, A., H. Zelelew and B. Muhunthan (2007). "Wavelet analysis of energy content in pavement roughness and truck dynamic axle loads." Transportation Research Record: Journal of the Transportation Research Board(2005): 153-159.

Papagiannakis, A. T., H. M. Zelelew and B. Muhunthan (2007). "A wavelet interpretation of vehicle-pavement interaction." International Journal of Pavement Engineering 8(3): 245-252.

Qin, Y., Z. Wang, C. Xiang, E. Hashemi, A. Khajepour and Y. Huang (2019). "Speed independent road classification strategy based on vehicle response: Theory and experimental validation." Mechanical Systems and Signal Processing 117(2019): 653-666.

Qin, Y., C. Xiang, Z. Wang and M. Dong (2017). "Road Excitation Classification for Semi-Active Suspension System Based on System Response."

Qin, Y., C. Xiang, Z. Wang and M. Dong (2018). "Road excitation classification for semi-active suspension system based on system response." Journal of vibration and control 24(13): 2732-2748.

Sayers, M. W. (1995). "On the calculation of international roughness index from longitudinal road profile." Transportation Research Record(1501).

Sayers, M. W., T. D. Gillespie and A. Queiroz (1986). The international road roughness experiment. Establishing correlation and a calibration standard for measurements. 
Sayers, M. W. and S. M. Karamihas (1998). "The little book of profiling." Ann Arbor: Transportation Research Institute, University of Michigan.

Shokouhi, P., N. Gucunski, A. Maher and S. Zaghloul (2005). "Wavelet-based multiresolution analysis of pavement profiles as a diagnostic tool." Transportation Research Record: Journal of the Transportation Research Board(1940): 79-88.

Spangler, E. B. and W. J. Kelly (1966). "GMR road profilometer-a method for measuring road profile." Highway Research Record.

Tai, Y.-c., C.-w. Chan and J. Y.-j. Hsu (2010). Automatic road anomaly detection using smart mobile device. conference on technologies and applications of artificial intelligence, Hsinchu, Taiwan, Citeseer.

Ward, C. C. and K. Iagnemma (2009). "Speed-independent vibration-based terrain classification for passenger vehicles." Vehicle System Dynamics 47(9): 1095-1113.

Wei, L. and T. Fwa (2004). "Characterizing road roughness by wavelet transform." Transportation Research Record: Journal of the Transportation Research Board(1869): 152-158.

Wei, L., T. Fwa and Z. Zhe (2005). "Wavelet analysis and interpretation of road roughness." Journal of Transportation Engineering 131(2): 120-130.

Wong, C. and K. Worden (2007). "Generalised NARX shunting neural network modelling of friction." Mechanical systems and signal processing 21(1): 553-572.

Wu, J.-J. (2000). "Simulation of rough surfaces with FFT." Tribology International 33(1): 47-58.

Zhang, Z., F. Deng, Y. Huang and R. Bridgelall (2015). "Road roughness evaluation using in-pavement strain sensors." Smart Materials and Structures 24(11): 115029.

Zhang, Z., F. Deng, Y. Huang and R. Bridgelall (2016). Field validation of road roughness evaluation using inpavement strain sensors. SPIE Smart Structures and Materials+ Nondestructive Evaluation and Health Monitoring, International Society for Optics and Photonics.

Zhang, Z., C. Sun, R. Bridgelall and M. Sun (2018). "Application of a Machine Learning Method to Evaluate Road Roughness from Connected Vehicles." Journal of Transportation Engineering, Part B: Pavements 144(4): 04018043. Zhou, J., P. Huang and F.-P. Chiang (2005). "Wavelet-based pavement distress classification." Transportation Research Record: Journal of the Transportation Research Board(1940): 89-98. 